\title{
Poétique et histoire littéraire : quand l'éthos donne le ton
}

Christine Le Quellec Cottier

\section{(2) OpenEdition \\ 1 Journals}

Édition électronique

URL : http://journals.openedition.org/edl/2511

DOI : $10.4000 /$ edl. 2511

ISSN : 2296-5084

Éditeur

Université de Lausanne

\section{Édition imprimée}

Date de publication : 15 décembre 2017

Pagination : 237-254

ISBN : 978-2-940331-66-6

ISSN : 0014-2026

Référence électronique

Christine Le Quellec Cottier, "Poétique et histoire littéraire : quand l'éthos donne le ton », Études de lettres [En ligne], 3-4 | 2017, mis en ligne le 15 décembre 2019, consulté le 17 décembre 2020. URL http://journals.openedition.org/edl/2511 ; DOI : https://doi.org/10.4000/edl.2511

(c) Études de lettres 


\section{POÉTIQUE ET HISTOIRE LITTÉRAIRE: QUAND L'ÉTHOS DONNE LE TON}

La réception des fictions proposant un imaginaire associé à une ou des Afriques - continentale ou diasporique - est encore fortement dépendante d'une histoire littéraire marquée par des catégories calquées sur le modèle français et souvent fixées par l'origine d'un auteur. Ces critères doivent se renouveler, car ils ne permettent plus de tisser un lien cohérent entre une production née, dans la cadre de la littérature francophone, au début du $\mathrm{XX}^{\mathrm{e}}$ siècle, et celle de notre $\mathrm{XXI}^{\mathrm{e}}$ siècle naissant. La proposition vise à modifier les repères de classification en exploitant la scène énonciative des textes, avant d'envisager un retour à l'axe du temps.

Au cœur d'Abidjan, il est un immeuble célèbre dont l'apparence est un leurre: sa structure solide existe pour moitié, mais l'autre, jamais terminée, donne l'illusion d'un bâtiment complet grâce à des planches peintes qui miment la façade extérieure; de nuit rien ne permet de percevoir la ruse, de jour, le regard curieux reconnaît le subterfuge. Cette fiction urbaine, métaphorique, me paraît à même de complexifier la reconnaissance de cette "Afrique telle qu'elle existe réellement " ${ }^{1}$, selon le souhait formulé par Christiane Albert à propos du devenir de la littérature francophone africaine, puisque de fait le réel n'y est plus qu'illusion tangible. Cette réalité aux allures multiples - construite, mimée; nocture, diurne; ... - est sans doute ce que propose constamment la fiction, car l'univers de mots qui bâtit un monde imaginaire n'a aucune obligation de référentialité. Le plus souvent sous-tendu par la notion de vraisemblance, le récit propose un espace où vivent des personnages dont

I. Ch. Albert, "Africanité et mondialisation chez les écrivains africains francophones", p. 63. 
l'existence offre une diversité - esthétique, politique ou culturelle - crédible. La ruse avec le réel est donc de l'ordre du subterfuge nécessaire et, du coup, le bâtiment d'Abidjan paraît l'heureuse métaphore du pouvoir de la fiction.

Cette créativité, illustrée par le bâtiment de la Radio-Télévision Ivoirienne, perd de sa pertinence lorsqu'il s'agit non plus d'apprécier la fiction elle-même, mais les catégories qui organisent notre analyse de la littérature francophone d'Afrique subsaharienne. Charpenté mais bancal, l'immeuble semble à l'image des ambiguïtés de l'histoire littéraire francophone dont les repères classificatoires n'ont cessé de bouger, quand le calque du modèle français s'avérait, aussi, problématique. Comment aujourd'hui motiver une histoire littéraire par le besoin de rendre compte d'une soit-disant " originalité africaine» ${ }^{2}$ ? Qu'est-ce donc? Quel est le lien, par exemple, entre Karim d'Ousmane Socé (1935) et Le terroriste noir de Tierno Monemenbo (2012)? Dans notre monde globalisé, les écrivains originaires d'Afrique veulent créer sans la contrainte, attendue par le monde éditorial européen, d'afficher une appartenance ou une référentialité culturelle les identifiant à une territorialité inaliénable. Et malgré ses ambiguïtés critiques et institutionnelles ${ }^{3}$, c'est bien cette tension qu'a voulu dénoncer le manifeste Pour une littérature-monde en français, publié en mars 2007 dans les colonnes du Monde, en refusant les ontologies essentialistes ${ }^{4}$ et l'éternelle autorité du "centre» envers la "périphérie». Les créations fictionnelles de notre extrême contemporain ne répondent plus aux images convenues que Xavier Garnier repérait en tant qu' «archaïsme régénérant» ou "laboratoire d'une hyper-modernité naïve " 5 . Ce socle a éclaté et notre monde globalisé place ses acteurs au cœur de nouvelles pratiques de négociation, formelles et symboliques, très souvent associées à un nouveau cosmopolitisme - marqué par les

2. M. Kane, "Sur l'histoire littéraire de l'Afrique subsaharienne francophone», p. 14.

3. Voir les analyses de V. Porra, «Malaise dans la littérature-monde (en français)» et de C. Ducournau, «Prologue: littérature francophone contre littérature-monde en français".

4. Il s'agit ici de la réception des textes mettant en scène un univers africain, et non des textes eux-mêmes.

5. X. Garnier, «Évolution actuelle des littératures africaines», p. 97. 
notions de migrance et d'hybridité ${ }^{6}$ - impliquant des espaces de horslieu ou de l'entre-deux où se négocient des appartenances multiples dont la langue est le médium; ces «migrances» touchent autant des mises en scène diasporiques que celles qui proposent un environnement continental identifié ou identifiable ${ }^{7}$.

Comment, dès lors, motiver une histoire littéraire qui éviterait les frontières essentialistes - se justifiant par l'origine subsaharienne de l'auteur - ou striée par des dates qui suivent les séquences historiques de la colonisation, puis de la décolonisation française ou belge, la production littéraire étant, dans ce cadre-ci, uniquement perçue dans sa dépendance à la présence de la langue française en Afrique ${ }^{8}$ Les critères de l'histoire littéraire française, tels que nous les a rappelés Alain Vaillant ${ }^{9}$, ne peuvent plus rendre compte des productions fictionnelles actuelles, forcément transnationales. Il paraît nécessaire de se détacher de ce modèle, tout comme il est désormais caduc de vouloir instaurer une histoire littéraire nationale dans chacun des pays d'Afrique, alors que les grandes aires de peuplements et de langues dépassent largement les démarcations frontalières ${ }^{10}$. Ainsi, à l'heure où la production artistique se matérialise dans un monde en réseau, il importe de proposer une alternative qui n'efface ni les singularités, ni l'axe du temps; notre but est de trouver

6. Selon l'anthropologue M. Agier (La condition cosmopolite, p. 91), "un hybride culturel [...] vit à la frontière de deux modèles différents de vie, sans savoir auquel des deux il appartient».

7. La «migrance» n'est donc pas en soit un déplacement géographique, mais convoque l'alternance des références, des codes langagiers et des cultures. À titre comparatif, les cinémas d'Afrique, qu'ils soient nigérians ou éthiopiens, rendent compte de ces cultures transnationales qui sont désormais partie prenante de toutes les appartenances revendiquées. Sur la notion de migrance, voir P. Ouellet, L'esprit migrateur.

8. Pour rappeler l'infondé d'une telle vision, S. Amedegnato cite le volume Essais d'histoire littéraire africaine d'Albert Gérard (Sherbrooke, 1984) qui analyse le champ littéraire africain en trois strates: la littérature écrite en langues européennes, celle écrite en langues africaines et celle nommée "performée», orale. Voir "Cinq remarques sur l'étiquette "littérature africaine" ", p. 376. Quant à la présente réflexion, elle se limite au champ de la littérature rédigée en langue française.

9. A. Vaillant, L'histoire littéraire, p. 41-43. L'auteur inventorie le principe d'une origine fondée sur une transmission orale; l'idée de la langue nationale unificatrice; la notion de mémoire littéraire; les figures de grands écrivains, dont l'existence et la reconnaissance sont étroitement liées à un rôle idéologique que le contexte a cautionné.

Io. M. Kane, "Sur l'histoire littéraire de l'Afrique subsaharienne francophone», p. $16 s q$. 
l'articulation qui maintienne un lien pertinent entre les productions contemporaines et celles du début du $\mathrm{XX}^{\mathrm{e}}$ siècle; il s'agit d'affirmer, comme l'a relevé Garnier, la cohérence d'une littérature née d'une «forte préoccupation culturaliste", alors que les productions d'aujourd'hui "porte[nt] un regard sur le monde, et en particulier sur l'Afrique, depuis un hors-monde» ${ }^{11}$.

L'ensemble constitué ne doit pas disparaître dans le global turn du moment, mais il faut questionner sa fabrication et son autonomie, puisque l'existence d'un champ littéraire africain reste très problématique et que les instances de consécration de langue française distribuant les clés du succès ${ }^{12}$ sont le plus souvent parisiennes. Si l'histoire littéraire, comme le notait Genette, est «une histoire des circonstances, des conditions et des répercussions sociales du fait littéraire", il faut aussi admettre, avec lui toujours, que "règne encore un préjugé qui est celui de [...] l'incompatibilité de l'étude synchronique et de l'étude diachronique, l'idée que l'on ne peut théoriser que dans la synchronie»" ${ }^{13}$.

Notre proposition veut donc traverser une centaine d'années de création en opérant une "révolution", puisque le lien existant entre les textes ne passe plus par l'identité auctoriale ou le déroulement chronologique. Il importe aussi de mettre à distance les polarités induites par la critique postcoloniale, rendant compte de la confrontation idéologique qui a marqué le $\mathrm{XX}^{\mathrm{e}}$ siècle: entre la justification initiale du champ postcolonial, visant à étudier toutes les cultures affectées par le processus impérialiste ${ }^{14}$ et notre monde contemporain, où les enjeux référentiels et symboliques se constituent sous le signe des nouveaux Empires ${ }^{15}$, la démarche a perdu de sa pertinence. Elle pourrait même être un piège, comme l'affirme Huggan pour qui la "plasticité du terme [...] suggère

II. X. Garnier, «Évolution actuelle des littératures africaines», p. 108.

I2. Sur ce sujet, lire C. Ducournau, La fabrique des classiques africains, passionnante histoire sociale collective des écrivains francophones africains en France depuis 1960.

I3. G. Genette, Figures III, p. 14 et 19.

I4. B. Mouralis («La critique des textes africains», p. 389) précise qu’une «littérature postcoloniale se développe dans une société affectée par une forme de domination coloniale européenne à partir du moment où cette production littéraire manifeste la volonté de s'opposer aux tendances de la littérature de la métropole, pour s'engager dans une voie propre».

15. "L'Empire devient un appareil décentralisé et déterritorialisé de gouvernement qui intègre progressivement l'espace monde", selon les historiens M. Hardt et A. Negri cités par Y. Clavaron, Poétique du roman postcolonial, p. 185. 
qu'il ne fonctionne pas seulement comme marqueur d'une résistance coloniale mais qu'il est aussi une étiquette promotionnelle " ${ }^{16} \mathrm{com}$ portant un risque de complicité avec l'impérialisme culturel. Huggan affirme que le postcolonial «brouille la frontière entre résistance et connivence " ${ }^{17}$ en créant des stratégies identitaires qui façonnent un nouveau canon, l'exotisme postcolonial. Cette ruse que Spivak nomme $l^{\prime}$ "essentialisme stratégique» ${ }^{18}$ renvoie à des intentionnalités d'auteur que notre démarche, par la prise en compte des textes avant celle de leur contexte, tend à évincer.

Il paraît donc judicieux de modifier les repères pour pouvoir constituer une nouvelle histoire littéraire qui s'intéresse d'abord aux formes de l'univers représenté, avant de tisser des liens avec le contexte social et politique de production et de réception. Ainsi, notre approche choisit de sérier les textes en fonction des types de voix qui mettent en scène une ou des Afriques, concrète ou diffractée, des voix qui perçoivent le monde depuis un imaginaire ou une expérience donnés pour africains. Ce projet alternatif s'organise à partir de l'instance énonciatrice du texte, l'éthos ${ }^{19}$, et prend donc comme point de convergence «une scène de parole qui n'est pas imposée par le type ou le genre de discours, mais instituée par le discours même» ${ }^{20}$, selon les propositions de Maingueneau. Cette scène est considérée comme le lieu où s'articulent l'œuvre et son contexte, et cette double fonction me permet de l'exploiter en tant que relais entre plusieurs niveaux d'analyse. Comme le postule Ruth Amossy dans La présentation de soi. Éthos et identité verbale, il s'agira de reconnaître comment le locuteur, soit la voix singulière qui le constitue, est en "prise sur des modèles culturels, sur un imaginaire social changeant dont elle se nourrit et qu'elle alimente en retour ${ }^{21}$ : ce double mouvement

I6. G. Huggan, «L'exotisme postcolonial», p. 289.

I7. Ibid., p. 290.

I8. G. Ch. Spivak développe ce concept dans Les subalternes peuvent-elles parler?.

19. L'éthos mobilise "tout ce qui, dans l'énonciation discursive, contribue à émettre une image de l'orateur à destination de l'auditoire. Ton de voix, débit de la parole, choix des mots et arguments, gestes, mimiques, regard, posture, parure, etc., sont autant de signes, élocutoires et oratoires, vestimentaires et symboliques, par lesquels l'orateur donne de lui-même une image psychologique et sociologique». G. Declercq, L'art d'argumenter - Structures rhétoriques et littéraires, Paris, Éditions Universitaires, 1992, p. 48; cité par D. Maingueneau, "L'ethos, de la rhétorique à l'analyse du discours», p. 1. 20. D. Maingueneau, Les termes clés de l'analyse du discours, p. 111.

21. R. Amossy, La présentation de soi, p. 7. 
cautionne une analyse qui se fonde sur des similitudes énonciatives pour rapprocher des textes, avant de mettre en perspective le temps et l'espace qui les ont vus naître.

Cette approche inductive ne se laisse pas berner par l'«Afrique inventée " ${ }^{22}$ dénoncée par Valentin Mubimbe, mais considère tout à la fois une topographie, espace concret, et une topologie, espace symbolique ${ }^{23}$ : l'Afrique est partout dans le monde, continentale, diasporique et cosmopolitique $^{24}$; elle a une existence concrète, mais elle est aussi une représentation symbolique qui travaille entre les langues et les cultures. Ces Afriques prennent place au cœur d'une histoire littéraire plus vaste, telle que McDonald et Suleiman l'ont proposée dans French global en 2010, pointant les connexions en langue française qui dépassent largement les frontières étatiques et permettent de rendre compte des transculturalités à l'œuvre dans les diverses communautés de langue française ${ }^{25}$. Cette option permet aussi d'envisager une littérature "franco-africaine ${ }^{26}$, affichant un lien, concret ou non, à un monde africain, quelle que soit l'origine de l'auteur ${ }^{27}$, et de croiser des temporalités, les textes regroupés n'ayant pas forcément paru à la même époque. Ce décalage temporel est pertinent pour mettre en perspective des contextes et des horizons d'attente et permettre, comme le réclamait Alain Ricard il y a plus de vingt ans, "une relecture de l'histoire littéraire d'Afrique noire, qui passerait par la prise en compte de l'histoire des formes, [qui] offrirait

22. Je cite le titre traduit de l'essai de V. Mubimbe, The invention of Africa: gnosis, philosophy and the order of knowlwdge. Disponible en ligne: <https://libcom.org/files/ zz_v._y._mudimbe_the_invention_of_africa_gnosis_pbook4you_1.pdf $>$.

23. S. Harel, Les passages obligés de l'écriture migrante, p. 28 sq.

24. Mot conçu à partir de "cosmopolitisme», désignant en sciences sociales la dimension dorénavant mondiale des relations sociales, le "cosmopolitique» implique une forme politique postnationale. Voir à ce sujet J. Y. Thériault «Au-delà du multiculturalisme: le cosmopolitisme?», p. 19.

25. Voir l'introduction à French global. Une nouvelle perspective sur l'histoire littéraire par Ch. McDonald et S. R. Suleiman, p. 13.

26. Selon le titre du colloque «La relation franco-africaine. Une nouvelle histoire politique et littéraire (1975-2015)». Université de Strasbourg (A. Mangeon et C. Grenouillet), 11-13 avril 2017.

27. Nous associons par exemple à ces ensembles le roman de Laurent Gaudé La mort du roi Tsongor, paru chez Actes Sud en 2003. 
l'occasion d'en finir avec les périodisations boiteuses et les points de vue abusivement négritudocentristes» ${ }^{28}$.

Le choix de la scène énonciative ${ }^{29}$ fait de l'éthos construit dans le texte le pivot de l'organisation des catégories proposées: la voix rend compte d'un sujet, c'est-à-dire d'une "conscience de soi »; celle-ci est considérée par les anthropologues ${ }^{30}$ comme un universel, puisqu'elle permet de "rendre raison des différences dans la manière d'habiter le monde et de lui donner un sens» ${ }^{31}$. Cette approche cautionne la "possibilité du sujet", non pas forcément en tant d'entités fixe ou stable ${ }^{32}$, mais plutôt celle d'une conscience de soi qui peut avoir des formes diverses, partielles et diffractées ${ }^{33}$. En articulant la réflexion à partir de l'éthos construit dans le texte, il est possible de repérer diverses typologies du "surgissement du sujet», processus que j’avais précédemment nommé "poétique de l'autodétermination » ${ }^{34}$, car caractéristique d'une littérature née en réaction à la domination coloniale.

Ainsi motivée, notre démarche propose six scènes énonciatives significatives ${ }^{35}$, à considérer comme des dominantes permettant de constituer une filiation entre le temps historique et aujourd'hui, soit une centaine d'années de création littéraire en français; elles sont organisées en fonction des caractéristiques d'une voix qui soit donne à lire une Afrique, soit

28. A. Ricard cité par J.-C. Blachère, Négritures, p. 244.

29. J.-M. Moura, dans Littératures francophones et théorie postcoloniale, a montré toute la pertinence de l'analyse de la situation énonciative, dans la fiction francophone.

30. Je fais ici surtout référence aux propositions de Philippe Descola dans Par-delà nature et culture.

3I. Ibid., p. 176.

32. Ce que l'africanisme colonial définissait comme la "personne», caractérisée par le rattachement à une culture, un lieu et une structure sociale. Voir M. Agier, La condition cosmopolite, p. 181.

33. Cela est d'importance quant au statut du sujet, comme le rappelle Anthony Mangeon à propos des travaux de Kagame et Wiredu: "Le cogito de Descartes (“je pense donc je suis") ne résiste pas [...] à sa traduction dans de nombreuses langues africaines». Voir A. Mangeon, La pensée noire et l'Occident, p. 124.

34. L'amorce de cette réflexion a paru en 2015: «La littérature francophone d'Afrique noire au prisme d'une poétique de l'autodétermination ".

35. Nous ne suivons donc pas les deux directions proposées par Achille Mbembe, dans Sortir de la grande nuit, pour penser la «reconstruction d'une identité africaine»: "l'une consiste en un effort de réenchantement de la tradition et de la coutume. L'autre procède par abstraction de la tradition, le souci principal étant l'émergence d'un soi moderne et déterritorialisé», cité par M. Agier, La condition cosmopolite, p. 151. 
une voix africaine qui perçoit le monde: ce double mouvement propose chaque fois un imaginaire africain, point de départ ou d'arrivée d'une relation avec l'ailleurs.

\section{Ethos cosmopolite}

Cette voix rend compte d'une «expérience culturelle plurielle, résumée en une unité de vie " ${ }^{36}$ qui porte son regard et son interprétation du monde. Il peut s'agir d'un narrateur, d'un personnage ou d'un je-lyrique mettant en scène une "migrance du moi » ${ }^{37}$, une instabilité qui rend compte d'une Afrique diasporique dont la présence ne peut se résumer aux images stéréotypées que le lecteur reconnaît depuis l'existence de la littérature coloniale ${ }^{38}$. Le constat est valable pour des textes comme Mémoires de porc-épic d'Alain Mabanckou (2006) ou Kétala de Fatou Diome (2006), romans qui réinvestissent très fortement des cultures africaines où les frontières du réel, telles que perçues en Occident, sont largement franchies, puisque la fable animale déjoue les attentes reconnues de la tradition orale, leitmotiv de ce qui a pu être considéré comme une "originalité africaine» et que le kétala a la forme d'une prosopopée, plaçant en miroir des traditions rhétoriques qui résonnent pour chacun des lecteurs. Les textes donnent à lire une vision du monde porteuse d'un savoir africain, mais associée à d'autres référents que l'intertextualité rend, entre autres, palpables; ils proposent des imaginaires où les traditions et les savoirs sont donnés à lire sur le même plan et avec la même légitimité que toute représentation, donc une normalité à saisir sans filtre. Les récits de fiction de notre extrême contemporain attestent d'une grande liberté de référents et de formes; ils pratiquent la mise à plat de l'Histoire, de la tradition et des stéréotypes, ce que l'on peut nommer de nouvelles formes d'engagement, puisqu'il ne s'agit pas, comme le voulait Mongo Beti durant les années 1960, d'afficher une appartenance et une identité revendicatrices africaines, mais de donner à lire, au niveau

36. Définition du cosmopolitisme littéraire proposée par J. Knebusch, Poésie planétaire, p. 37.

37. P. Ouellet, L'esprit migrateur, p. 26 sq.

38. Dans celle-ci l'éthos africain est objet et non sujet, ce qui justifie son exclusion de notre typologie. Batouala de René Maran, paru en 1921, nous paraît à ce titre significatif d'un processus de transformation. 
individuel et collectif, ce qui questionne ces mêmes appartenances, non sans ironie:

[...] le trépassé comprit ce que l'on attendait de lui d'autant que, dans ses propos, le féticheur ajouta «ne nous fait surtout pas honte devant ces Blancs qui sont venus de loin et qui prennent nos coutumes pour de la simple rigolade», le cadavre ne se fit pas prier deux fois [... ${ }^{39}$

À cet égard, Le terroriste noir de Monenembo (2012) déjoue les attentes trop souvent liées à un nom d'auteur africain devant «représenter l'Afrique», puisque la ruse narrative consiste à faire parler Germaine Tergonesse, Vosgienne de 80 ans, qui conte sa propre expérience, partielle et distante, d'une Afrique incarnée par le résistant Addi Bâ ${ }^{40}$ : la perspective choisie par le récit rend palpable la nécessaire réévaluation des critères de classement, afin de ne pas réduire cette inventivité fictionnelle à une vague rubrique «Mutations et perspectives» ${ }^{41}$.

\section{Ethos de genre}

Ces voix impliquent la reconnaissance de «comportements sociaux et attentes de rôles définissant à chaque époque les rôles respectifs, répétant [...] la normalité» ${ }^{42}$ : les implicites sont mis à jour et questionnés, en plaçant dans la fiction un regard particulier qui réévalue son environnement. Cet éthos est particulièrement significatif dans les récits où les personnages féminins déploient leur regard sur la société patriarcale qui les conditionne ou dévoilent les effets de cette identité construite dans un univers étranger. On pense bien sûr à Ramatoulaye dans le roman épistolaire Une si longue lettre de Mariama Bâ (1979), qui a fondé une lignée de voix féminines revendicatrices. La dernière lettre de Salla Dieng, paru en 2008, en est un riche écho, passant d'un Sénégal natal à une prison française, transformant la mère au foyer en immigrée méprisée, donnant vie à un destinataire masculin plutôt qu'à l'amie de longue date:

39. A. Mabanckou, Mémoires de porc-épic, p. 144.

40. Ch. Le Quellec Cottier, «Histoire et histoire littéraire», p. 28.

4I. M. Kane, "Sur l'histoire littéraire de l'Afrique subsaharienne francophone», p. 25.

42. A. Oberhuber, «Un genre incertain, après tout», p. 310. 
Mon Dieu, Serge!

Que la société est cruelle et intolérante!

En plus de l'exil dans un pays qui différait tellement de celui qui m’avait vu naître, je connaissais la pointe plus acérée et létale de la ségrégation des miens.

Oui, même dans ce pays où les mots «liberté, égalité et fraternité» n'étaient pas qu'illusion, apparence, j’ai connu l'enfer de cette double ségrégation qui pointait mon enfant sans père du doigt, cette «bâtarde qui attire la poisse ${ }^{43}$.

Nées quarante ans après les premières voix masculines affirmant une identité africaine au milieu du brouhaha colonial, les voix féminines peuvent aussi être associées aux voix des enfants-soldats, subalternes d'un monde qui n'est plus apte à les protéger: devenus symboles d'une société défaite, ils sont le pendant de «l'enfant-terrible» des contes qui, lui, mettait en garde contre l'hybris individuelle, puissance dévastatrice pour la communauté. Les voix des enfants-soldats, nées des guerres civiles et de génocides, sont la trace inversée du lien à un espace et une culture: la communauté-parentelle est désormais inexistante et l'hybris destructrice est portée par un environnement toxique, meurtrier que rien ne semble pouvoir renverser. La violence des scénographies permet aussi d'envisager un nouveau groupe au sein de ces voix démultipliées par leur différence «genrée», celui des LGBT de la communauté arc-en-ciel:

Je n’ai jamais voulu te faire de mal Mémoria, tu peux me croire. [...] En réalité les femmes ne m'attirent pas, sinon je ne vois pas comment j’aurais pu résister à une fille aussi magnifique que toi. Et tu te trompes au sujet de Tamara.

[...] Pervers sadiques, libres de jouir de leur domination ou homosexuels honteux, cachés derrière l'image hyper-virile de l'armée, les trois sous-officiers savaient qu'ils avaient plus à craindre d'une attaque d'extraterrestres que d'un éventuel soulèvement des bleus. En outre, Tamsir [Tamara] ne pouvait compter sur la solidarité de ses collègues: ces machos nourrissaient une haine farouche des homosexuels et considéraient les sévices qu'il endurait comme un châtiment mérité ${ }^{44}$.

43. S. Dieng, La dernière lettre, p. 83 sq.

44. F. Diome, Kétala, p. 90 sq. et 101 sq. 


\section{Éthos d'opposition et éthos démultiplié}

Les voix associées par cette typologie de scènes énonciatives proposent un discours d'opposition qui peut être perçu de deux façons significatives; la voix porte des enjeux qui impliquent une confrontation idéologique au niveau thématique (opposition) et/ou la forme de son discours crée une confrontation esthétique au niveau linguistique (démultiplication). La scénographie postcoloniale dénonce la situation de domination coloniale ou postcoloniale, par des discours qui touchent autant par l'ironie, le grotesque ou encore la naïveté d'une voix qui ne sait interpréter ce qu'elle voit. On peut penser autant au Pauvre Christ de Bomba (Mongo Beti, 1956) qu'à Une vie de boy (Ferdinand Oyono, 1956), à la poésie de Tchicaya U Tamsi criant dans Le ventre sa révolte, personnelle et politique. Mais cette opposition est déjà active avec les voix des pères fondateurs de la Négritude qui, dans leur poésie, ont fait exister le sujet africain et le sujet colonial en tant que sujet révolté. Au début de ces années 1930, les auteurs écrivent "dans la langue » ${ }^{45}$, en maintenant un ordre du discours français, indépendamment de tout écart stylistique. La "démultiplication" se matérialise trente ans plus tard dès Les soleils des indépendances (A. Kourouma, 1968) et Le devoir de violence (Y. Ouologuem, 1968) qui présentent des scènes énonciatives "hors de la langue", c'est-à-dire proposant une mise en perspective nouvelle de la langue française: «l'écriture s'origine dans l'interférence linguistique [et] le style ne traduit plus une vision du monde, mais une vision distancée de la langue. On n'a pas un écart dans la langue mais un écart de la langue elle-même» ${ }^{46}$. La prolifération contestataire de toute norme déconstruit les discours d'autorité, par exemple grâce à la polyphonie, tel Le Pleurer-Rire d'Henri Lopes (1982) ou par la carnavalisation grotesque de la langue, du discours, du vocabulaire et de la syntaxe que le théâtre et les romans de Sony Labou Tansi ravagent. Les voix commentent des pays imaginaires dont les attributs renvoient à un savoir partagé sur l'Afrique, à une époque de dictature qui a marqué l'histoire de la Guerre froide, des traîtrises sans fin dont le matériau langagier devient le support même. Dans Les soleils des indépendances d'Ahmadou Kourouma (1968), la diégèse, portée par un narrateur qui se dit malinké, relayant les

45. X. Garnier, «La littérature africaine francophone», p. 238.

46. Ibid., p. 240. 
paroles et pensées de ses personnages, permet de comprendre le décalage spirituel d'une génération qui s'est cru porteuse d'une autorité éternelle, alors qu'elle n'a pas su se montrer digne du monde nouveau à construire. Dans un univers de "bâtardises", la langue de Kourouma met les failles sociales et politiques en scène non pour prôner une "hybridité» renouvelée, mais pour dénoncer une inadéquation magistrale entre un univers de pensée et sa matérialité quotidienne:

«Regardez Fama! Regardez le mari de Salimata! Voyez-moi, fils de bâtards, fils d'esclaves! Regardez-moi partir!» Des cris de stupeur échappèrent aux voyageurs. [...] Et comme toujours dans le Horodougou en pareille circonstance, ce furent les animaux sauvages qui les premiers comprirent la portée historique du cri de l'homme, du grognement de la bête et du coup de fusil qui venaient de troubler le matin ${ }^{47}$.

\section{Éthos mémoriel}

Cette voix propose l'expérience collective en actualisant un temps historique entrant en résonance avec le moment d'énonciation, ou de publication. Elle dit le temps de l'Histoire et place les personnages au seuil de destinées qui rendent compte d'une culture ou d'appartenances. Avec des scénographies diverses s'associent à cette catégorie, par exemple La saison de l'ombre de Miano (2013), Monnè, outages et défis de Kourouma (1990), Peuls de Monenembo (2004) ou le très récent Confidences de Lobe (2016) qui prête à Ma Malanga, une Camerounaise âgée, le récit très oralisé des années de lutte pour l'indépendance qu'elle a traversées aux côtés de Um'Niobe. Parmi ces textes pourrait être placé Reine Pokou, concerto pour un sacrifice (Tadjo, 2004) dont le caractère mémoriel est acquis, puisqu'il présente une légende baoulé fondatrice de la Côte d'Ivoire actuelle. Mais, simultanément, le texte propose des variantes à la légende qui déjouent la mémoire et son interprétation: par ces voix multiples qui questionnent la portée de la tradition et les alternatives à une histoire placée sous le signe du sacrifice, nous plaçons ce récit parmi l'ensemble des éthos "démultipliés». Comme le relevait Garnier à propos du rapport distancié à la langue - française -, la force de cette littérature née

47. A. Kourouma, Les soleils des indépendances, p. 191 sq. 
d'un paradoxe pragmatique ${ }^{48}$ tient aussi au fait que les textes de fiction s'associent à un héritage culturel, mais le questionnent simultanément par des stratégies narratives, des ruses formelles.

\section{Éthos de la présence}

Cette catégorie regroupe les récits donnant voix à un sujet africain qui rend compte d'un univers africain. La voix narrative ou lyrique se veut souvent descriptive, et son discours, même s'il semble imitatif, est déjà une forme d'autonomie, comme l'affirmait Blachère pour les premiers récits écrits en français par des Africains: «En situation coloniale, écrire c'est déjà protester. Copier un modèle, c'est d'une certaine manière abolir les barrières et les hiérarchies " ${ }^{49}$. L'univers africain proposé est vu par celui qui en partage la connaissance et l'imaginaire, il rend compte d'une expérience qui souvent se veut témoignage. Pensons aux romans publiés dans les années 1920, tels Les trois volontés de Malick (A. M. Diagne, 1920), Force bonté (Bacary Diallo, 1926) ou L'esclave (Felix Couchoro, 1929), mais aussi aux romans d'apprentissage - dont la forme a gardé la mémoire des contes à valeur didactique et des récits épiques portant le devenir d'un être d'exception - qui ont marqué le début du XX $\mathrm{XX}^{\mathrm{e}}$ siècle, par exemple, Karim d'Osmane Socé (1935) ou L'enfant noir de Camara Laye, paru en 1956. Chacune de ces voix veut affirmer une appartenance et une origine que le temps colonial s'est employé à nier.

Classées ainsi, ces voix souvent datées permettent aussi de différencier les enjeux des «représentations africaines»: L'enfant noir semble le point limite des voix accomplies, proposées par le genre du roman d'apprentissage, car la même année paraît Une vie de boy dans lequel le devenir positif ne peut plus se matérialiser. La déchéance du boy Toundi, sa fuite, ses blessures et son corps putréfié attestent d'un éparpillement destructeur qui rapproche sa voix de celle des enfants-soldats et donc d'un «éthos genré».

48. J.-M. Moura, Littératures francophones et théorie postcoloniale, p. 76 sq. : «dans la mesure où ce que dit l'énoncé (sa forme et son contenu, soumis aux modèles français) entre en contradiction avec ce que montre son énonciation, à savoir: une prise de parole indigène reconnue comme telle».

49. J.-C. Blachère, Négritures, p. 10. 
Les séquences proposées délimitent des ensembles de textes qui résonnent de voix complices, proches les unes des autres par leurs enjeux et leurs formes. Ces regroupements défient l'axe linéaire de la chronologie en associant des ouvrages parus à des dates différentes; ce constat me semble de bon augure pour repenser la lecture d'une histoire littéraire dont les prémices furent conditionnées par la contrainte coloniale. Cent ans plus tard, comme d'autres littératures de langue française, l'univers africain représenté dans la fiction, choisi comme motif et pratique langagière, modifie les repères, mais permet de traverser ce siècle de création en gardant le fil d'un devenir au sein de la langue française, amorcé par des voix qui cherchaient leur propre autodétermination, à celles d'aujourd'hui, affranchies, qui se lisent en phase avec des imaginaires du monde entier, portant simultanément plusieurs cultures dont le point de contact est, pour la démarche qui est la nôtre, la langue française. Les propositions faites, à notre avis pertinentes pour toutes les littératures qui ont eu à se positionner face au champ littéraire français, veulent dépasser les polarités qui conditionnent encore nos références critiques. Aborder les textes en observant les voix qui offrent un regard sur ou depuis l'Afrique permet de construire une histoire littéraire dont les séquences, à l'image des textes eux-mêmes, questionnent le rapport au temps, à l'espace et aux identités assignées.

Les propositions faites se voudraient une judicieuse façon de sortir d'une impasse critique, celle de l'histoire littéraire actuelle. Rappelonsnous que si ruse il y a, sa capacité à leurrer, associée à l'ancienne mètis grecque, reste en Afrique de l'Ouest une marque d'intelligence pratique, un stratagème positif... Face à l'immeuble de la RTI, chantier bancal de l'histoire littéraire, imaginons donc sa reconversion!

Christine Le Quellec Cottier

Université de Lausanne 


\section{BIBLIOGRAPHIE}

Agrer, Michel, La condition cosmopolite, Paris, La Différence, 2013.

Albert, Christiane, "Africanité et mondialisation chez les écrivains africains francophones", in Figures croisées d'intellectuels, éd. par A. Kouvouama, A. Gueye, A. Piriou, A.-C. Wagner, Paris, Karthala, 2007, p. 57-66.

Amedegnato, Sénamin, "Cinq remarques sur l'étiquette "littérature africaine" ", in Écritures et mythes. L'Afrique en question, éd. par S. K. Gbanou, S. Amedegnato, Bayreuth, Bayreuth African Studies Series, 2006, p. 373-394.

Amossy, Ruth, La présentation de soi. Éthos et identité verbale, Paris, PUF, 2010.

Blachère, Jean-Claude, Négritures. Les écrivains d'Afrique noire et la langue française, Paris, L'Harmattan, 1993.

Clavaron, Yves, Poétique du roman postcolonial, St-Etienne, PUSE, 2011.

Descola, Philippe, Par-delà nature et culture, Paris, PUF, 2006.

Dieng, Salla, La dernière lettre, Paris, Présence africaine, 2008.

Diome, Fatou, Kétala, Paris, J'ai lu, 2007.

Ducournau, Claire, "Prologue: littérature francophone contre littérature-monde en français", in La fabrique des classiques africains, Paris, CNRS Éditions, 2017, p. 31-88.

Garnier, Xavier, "La littérature africaine francophone: une affaire de style?», in Les études littéraires francophones: états des lieux, éd. par L. D’Hulst, J.-M. Moura, Lille, Travaux du Conseil Scientifique de l'Université Charles-de-Gaulle-Lille 3, 2003, p. 235-244.

—, "Évolution actuelle des littératures africaines», Cahiers de l'Association internationale des études françaises, 59 (2007), p. 97-108.

Genette, Gérard, Figures III, Paris, Seuil, 1972 (Poétique).

Harel, Simon, Les passages obligés de l'écriture migrante, Montréal, XYZ, 2005.

Huggan, Graham, "L'exotisme postcolonial. Salman Rushdie et le Booker des Booker", in Postcolonial studies: modes d'emploi, éd. 
par Collectif Write Back, Lyon, Presses universitaires de Lyon, 2013, p. 287-301.

Hutcheon, Linda, Valdés, Mario J., Rethinking literary history. A dialogue on theory, New York, Oxford University Press, 2002.

Kane, Mohamadou, "Sur l'histoire littéraire de l'Afrique subsaharienne francophone", Etudes littéraires: L'institution littéraire en Afrique subsaharienne francophone, 24/2 (automne 1991), p. 9-28 et en ligne: <https://www.erudit.org/fr/revues/etudlitt/1991-v24-n2etudlitt2245/500964ar/>.

Knebusch, Julien, Poésie planétaire, Paris, Presses Sorbonne-Nouvelle, 2012.

Kourouma, Ahmadou, Les soleils des indépendances, Paris, Seuil, 1970.

Le Quellec Cottier, Christine, «La littérature francophone d'Afrique noire au prisme d'une poétique de l'autodétermination", in 1913: cent ans après. Enchantements et désenchantements, éd. par Colette Camelin, Marie-Paule Berranger, Paris, Hermann, 2015, p. $127-144$.

—, "Histoire et histoire littéraire: l'autodétermination des romans de Tierno Monenembo", Dalhousie French studies, 108 (2017), p. 33-42.

Mabanckou, Alain, Mémoires de porc-épic, Paris, Seuil, 2007.

Maingueneau, Dominique, "L'ethos, de la rhétorique à l'analyse du discours", version raccourcie et légèrement modifiée de "Problèmes d'ethos ", Pratiques, 113-114 (juin 2002), disponible en ligne: $<$ http://dominique.maingueneau.pagesperso-orange.fr/pdf/Ethos. $\mathrm{pdf}>$.

—, Les termes clés de l'analyse du discours, Paris, Seuil, 2009 [1996].

Mangeon, Anthony, La pensée noire et l'Occident. De la bibliothèque coloniale à Barack Obama, Cabris, éd. Sulliver, 2010.

McDonald, Christie, Suleiman, Susan Rubin (éds), French global. Une nouvelle perspective sur l'histoire littéraire, Paris, Garnier, 2014 [2010].

Moura, Jean-Marc, Littératures francophones et théorie postcoloniale, Paris, PUF, 2007 [1999].

Mouralis, Bernard, "La critique des textes africains. Entre théorie et science", in Les chemins de la critique africaine, éd. par G. B. Madébé, S. Mondadori, S. R. Renombo, Paris, L'Harmattan, 2012, p. 379-394. 
Mubimbe, Valentin, The invention of Africa: gnosis, philosophy and the order of knowlwdge, Bloomington/London, Indiana University Press, 1988.

Oberhuber, Andrea, "Un genre incertain, après tout", in Un certain genre malgré tout. Pour une réflexion sur la différence sexuelle à l'œuvre dans l'écriture, éd. par C. Mavrikakis, P. Poirier, Québec, Nota Bene, 2006, p. 303-316.

Ouellet, Pierre, L'esprit migrateur, Montréal, VLB Editeur, 2005.

Porra, Véronique, "Malaise dans la littérature-monde (en français): de la reprise des discours aux paradoxes de l'énonciation", Recherches et travaux, 76 (2010), p. 109-129 et en ligne: <https:// recherchestravaux.revues.org/411>.

SpIvak, Gayatri Chakravorty, Les subalternes peuvent-elles parler?, traduction de J. Vidal, Paris, Éditions Amsterdam, 2009 [1988].

Thériault, Joseph Yvon, «Au-delà du multiculturalisme: le cosmopolitisme?", Sociologie et sociétés, 44/1 (2012), p. 17-33.

Vailllant, Alain, L’histoire littéraire, Paris, Armand Colin, 2010. 
